\title{
Design and characteristics of series-series and series-parallel topologies fed from constant-voltage fixed-frequency supply
}

\author{
KUNWAR ADITYA \\ General Motors-Automotive Center of Excellence (GM-ACE), Faculty of Engineering and Applied Science, \\ Department of Electrical, Computer and Software Engineering, University of Ontario Institute of Technology, \\ Oshawa, ON L1H 7K4, Canada \\ e-mail: kunwar.aditya@uoit.ca
}

MS received 17 June 2015; revised 1 September 2016; accepted 1 October 2016

\begin{abstract}
Characteristics of series-series and series-parallel topologies when fed from a constant-voltage fixed-frequency supply have been studied. Characterization helps understanding the fundamentals of the topologies and helps in selection of particular topology for a particular application. For studying the characteristics a prototype of IPT coils has been developed in the laboratory. Parameters of the coil have been found using finite-element analysis software JMAG. Characteristics have been simulated in MATLAB Simulink and results obtained have been compared with those obtained from the hardware implementation of the characteristic. It has been shown in this paper that series-series topology has ideal current-source characteristics and series-parallel topology has ideal voltage-source characteristics.
\end{abstract}

Keywords. Inductive power transfer; finite-element analysis; transfer function; circuit synthesis; battery charging.

\section{Introduction}

In recent years wireless power transfer (WPT) for charging of electric vehicles (EVs) has gained worldwide attention. At the heart of this wireless power transfer is two coils separated by air gap [1]. Way side coil, also called transmitter coil or primary coil, is installed on the ground, whereas pick-up or secondary coil is installed underneath the vehicle. Due to the absence of any galvanic contact between the primary and secondary, the charging process becomes automated, convenient and unaffected by dirt, chemicals and the weather, therefore making it robust, reliable, safe and free from inconvenience of traditional plugs $[2,3]$. In literature this method of power transfer has also been referred to as WPT, contactless power transfer (CPT), contactless energy transfer (CET), inductively coupled power transfer (ICPT) and inductive power transfer (IPT) [4]. In this paper, the term IPT will be used hereafter for consistency. To allow for inconsistency in the road surface and better clearance between the road and vehicle, a large air gap is required between primary and secondary coils, due to which coupling is poor and leakage inductance is large compared with mutual inductance. Therefore this system of power transfer can be put into a loosely coupled system as compared with transformer and induction motors, which are classified as a closely coupled system [5].
Poor coupling in loosely coupled systems leads to poor transfer of power. To compensate the effect of poor coupling and leakage inductance, capacitive compensation in primary and secondary windings is required. For this purpose, a capacitor can be connected in either series or parallel of both windings giving a total of four topologies namely SeriesSeries (SS) topology, Series-Parallel (SP) topology, Parallel-Series (PS) topology and Parallel-Parallel (PP) topology [6]. Since the capacitor is made to resonate with self-inductance of primary and secondary coils it is more appropriate to call it a resonant inductive coupling rather than merely inductive coupling [7]. Resonant inductive coupling, also called as electrodynamic induction, was used as early as 1894 by Nikola Tesla to wirelessly light up phosphorescent and incandescent lamps at the 35 South Fifth Avenue laboratory, and later at the Houston Street laboratory in New York City [8]. In literature it has been suggested that the choice of topology depends upon application. There has been research to find out the most suitable topology for particular applications such as battery charging [9]. For example for charging application the topology that can act as a constant current source will be a good choice [10]. However there is hardly any paper on characteristics of these topologies that gives definite relation for current transfer ratio and voltage transfer ratio of these topologies. They have been mentioned in papers but there is no proof of concept presented [11]. 
For EV battery charging, the charging process usually consists of four main stages: trickle charging, constant current (CC) charging, constant voltage (CV) charging and charge termination [12]. A CC charging stage and a CV charging stage, as shown in figure 1, is the preferred charging algorithm for $\mathrm{Li}$-ion battery chemistry [13]. During the CC charging stage, the battery is charged at CC until the specified peak voltage of battery cells is reached. During the $\mathrm{CV}$ charging stage, the charging takes place at CV. At the end of CC charging stage, the battery cell voltage is at the specified peak value and this voltage is maintained across the battery cell throughout the $\mathrm{CV}$ charging stage. Hence for charging application the topology that can act as a CC source or as CV source will be a good choice.

In this paper, analysis of SS and SP topologies has been presented using two-port network theory. From two-port parameters, a steady state transfer function between input parameters (i.e., input voltage and current) and output parameters (i.e., output voltage and current) has been established. The aim is to establish the trend of variation of output parameters when the primary is supplied by a CV source of fixed frequency nature. For this purpose a prototype of IPT coils has been fabricated in the laboratory. The primary of IPT has been fed from a CV source of fixed frequency for both topologies. This paper has been arranged in the following manner: in section 2, design of IPT coils used in the analysis has been discussed; in section 3, analysis of SS topology has been presented; in section 4, analysis of SP topology has been presented; in section 5, analysis has been supported by simulation and experimental results and section 6 , concludes the paper.

\section{Design of IPT coils}

For the proof of concept of theories presented in this paper a prototype of coreless IPT coils has been developed in the laboratory as shown in figure 2. In high-frequency

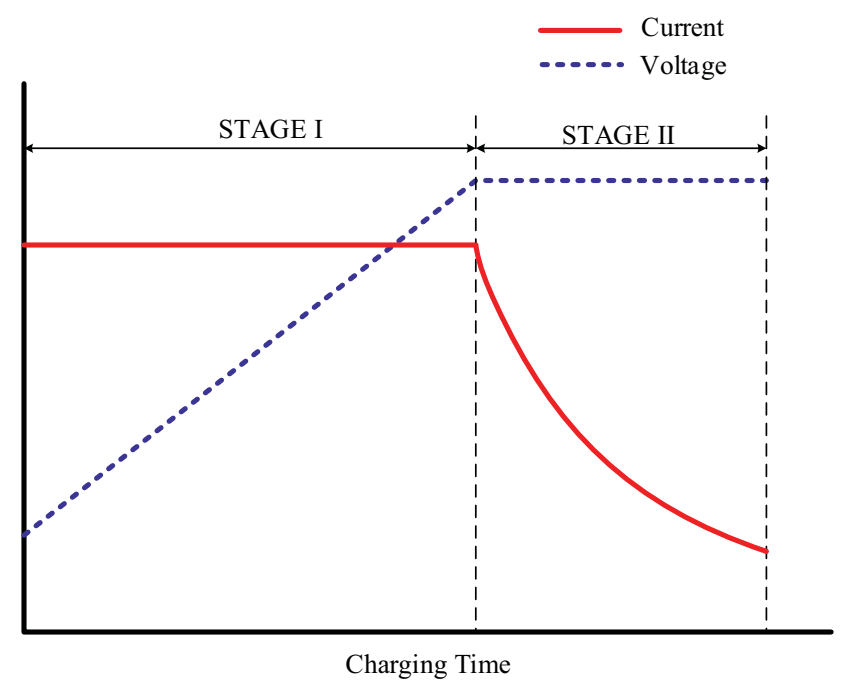

Figure 1. Typical charging profile of Li-ion batteries.

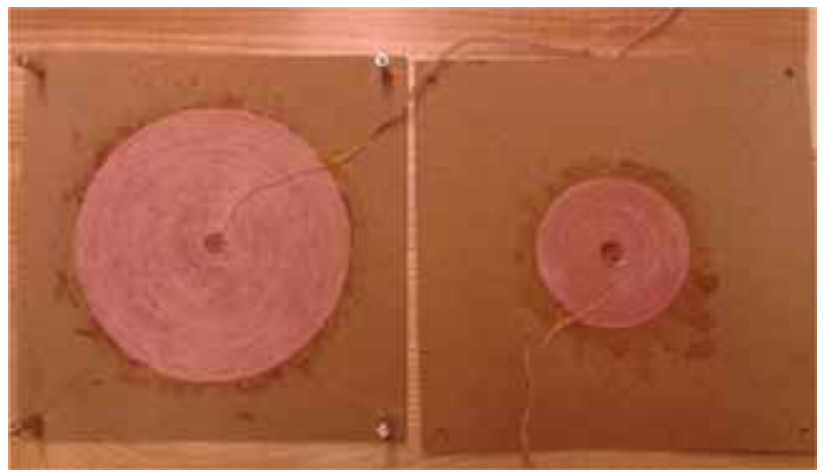

Figure 2. Primary and secondary coils.

Table 1. Coils specifications.

Primary coil

Wire diameter: $1.2446 \mathrm{~mm}$

Coil inner diameter: $1 \mathrm{~cm}$

Coil outer diameter: $15 \mathrm{~cm}$

Number of turns: 50

Secondary coil

Wire diameter: $1.2446 \mathrm{~mm}$

Coil inner diameter: $1 \mathrm{~cm}$

Coil outer diameter: $7.5 \mathrm{~cm}$

Number of turns: 25

operation, equivalent series resistance (ESR) of wire and therefore copper loss increases due to the skin effect and proximity effects. To mitigate skin effect and proximity effect, braided enamelled conductors, also known as litz wire, can be used for making the coils. Therefore, litz wire 90/38 SPN SN (90 strands, 38 AWG each strand) has been used to build the coils. Table 1 gives the specification of primary and secondary coils.

Parameters of the coils such as mutual inductance and self-inductance were measured experimentally and were verified using a finite-element analysis (FEA) software named JMAG. A simpler way of calculating mutual inductance between two coils is by measuring the open circuit voltage across one coil if a current of known magnitude and frequency is flowing in another coil, i.e., if ' $V_{\mathrm{oc}}$ ' is open circuit voltage in a coil and ' $I_{1}$ ' is the current of known magnitude and frequency ' $\omega$ ' flowing in another coil then mutual inductance ' $M$ ' is given by

$$
M=\frac{V_{\mathrm{oc}}}{\omega I_{1}} .
$$

Self-inductance and resistance of any coil can be calculated by measuring the current flowing in the coil when an alternating voltage of known frequency is applied to the coil [14]. To separate resistance from inductance, phase difference between voltage and current can be calculated using an oscilloscope. Experimental set-up for measuring self-inductance of secondary has been shown in figure 3 . 


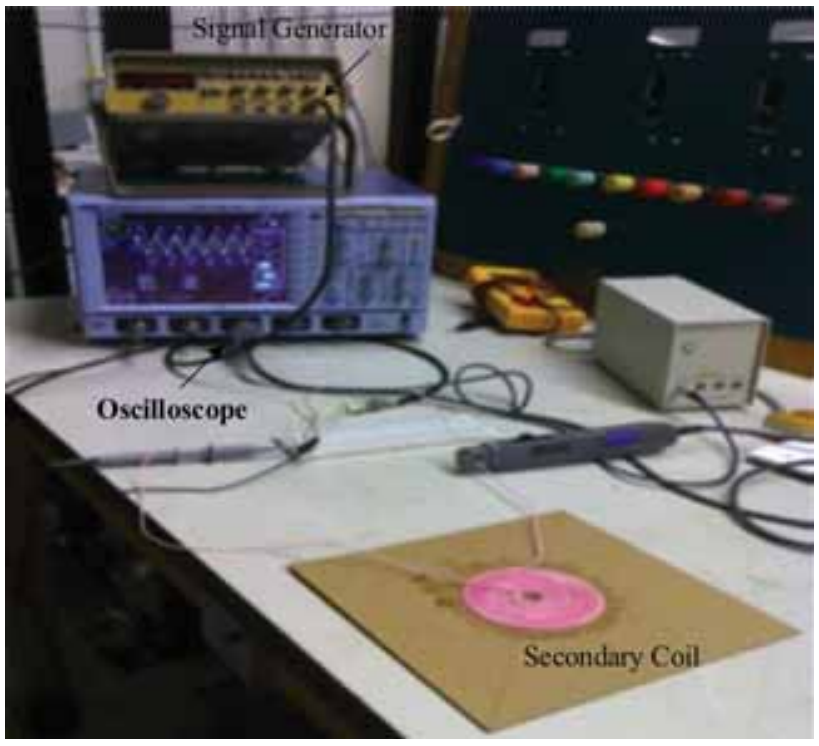

Figure 3. Set-up for calculating self-inductance of secondary coil.

For the verification of experimental results, coils have been modelled in JMAG software. A 3D 1/2 model using magnetic field/frequency analysis and 2D axisymmetric model of the coils were created and were compared with experimental results. As mentioned in table 1 the diameter of the litz wire used was $1.2446 \mathrm{~mm}$ and it consist of 90 strands, each having its own insulation, due to which it was practically impossible to model each and every strand individually. To tackle this problem the coils were modelled as a single solid region as an approximation to the litz wire and to make current density uniform throughout the cross-section of wire 'allow eddy currents' is not selected in material settings of the coil in JMAG. This approximation works due to fact that litz wire makes the current density uniform throughout the cross-section of the conductor. Figure 4 shows the JMAG model of the coils for calculating mutual inductance between coils at $20 \mathrm{kHz}$ and $25 \mathrm{~mm}$ air gap.
Table 2 compares the parameters obtained experimentally with the values obtained through $2 \mathrm{D}$ and $3 \mathrm{D}$ JMAG analysis at $20 \mathrm{kHz}$ and $25 \mathrm{~mm}$ air gap.

From table 2 one can observe that self-inductance and mutual inductance obtained with experiment and JMAG are in close agreement. The 3D model is more accurate and close to experimental results since magnetic flux in the axial direction is ignored in the 2D model but it is present in the 3D model. In this paper, experimental results were used in the simulation.

\section{Characteristics of SS topology}

Figure 5 gives the IPT coils with SS compensation.

The subscripts 'p' and 's' represent primary and secondary side, respectively. Necessity of compensation has already been discussed in Aditya and Williamson [2, 3]. Value of primary and secondary capacitance at resonance frequency $\left(\omega_{0}\right)$ is given by Stielau and Covic [15]

$$
\begin{gathered}
C_{\mathrm{p}}=\frac{L_{\mathrm{s}} C_{\mathrm{s}}}{L_{\mathrm{p}}} \\
C_{\mathrm{s}}=\frac{1}{\omega_{o}^{2} L_{\mathrm{s}}} .
\end{gathered}
$$

Since IPT coils are air cored they behave as a linear transformer and therefore linear circuit analysis can be

Table 2. Parameters of coils at $20 \mathrm{kHz}$ and $25 \mathrm{~mm}$ air gap.

\begin{tabular}{lccc}
\hline Parameters & Experimental & 2D JMAG & 3D JMAG \\
\hline$L_{\mathrm{p}}(\mu \mathrm{H})$ & 142.12 & 137.73 & 140.91 \\
$L_{\mathrm{s}}(\mu \mathrm{H})$ & 22.25 & 21.75 & 22.01 \\
$M(\mu \mathrm{H})$ & 31.4 & 33.35 & 32.24 \\
$R_{\mathrm{p}}(\Omega)$ & 0.2244 & 0.2244 & 0.2244 \\
$R_{\mathrm{s}}(\Omega)$ & 0.1174 & 0.1174 & 0.1174 \\
\hline
\end{tabular}

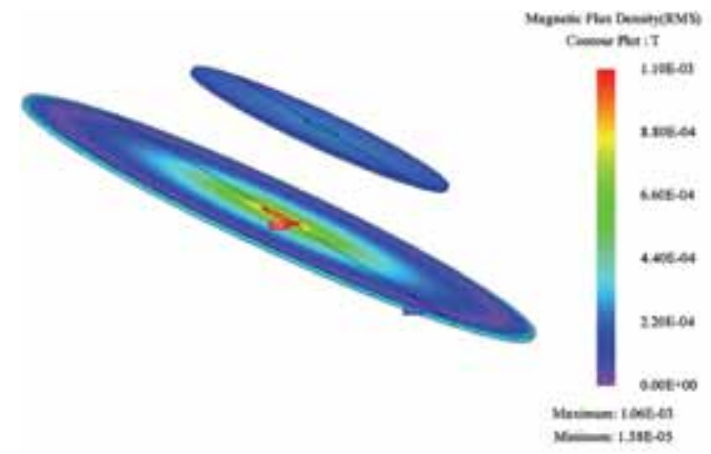

(a)

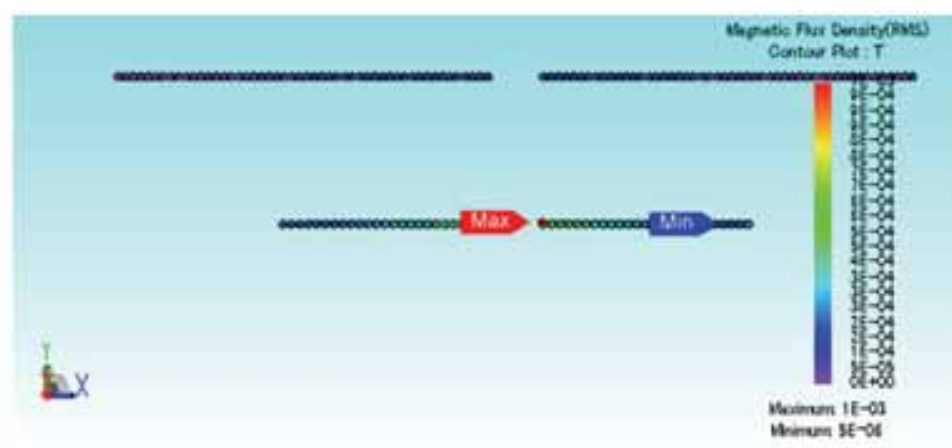

(b)

Figure 4. JMAG model for calculating mutual inductance: (a) 3D model, isometric view and (b) $2 \mathrm{D}$ model. 

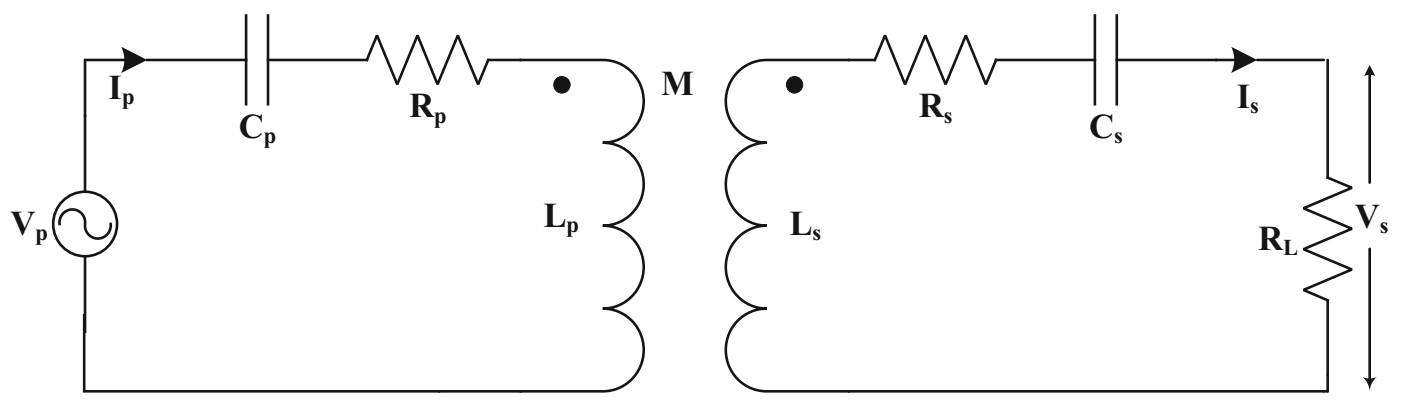

Figure 5. SS compensated IPT topology.

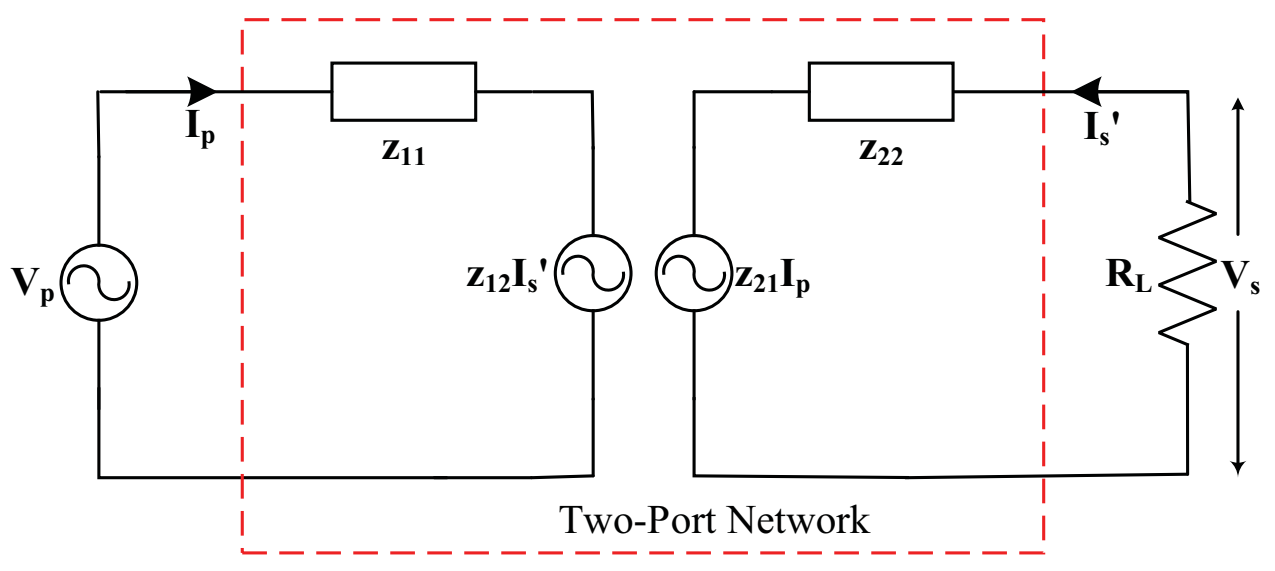

Figure 6. Two-port model of SS topology.

applied in this case. Figure 6 gives the two-port network circuit of the circuit shown in figure 5 .

The circuit inside the red box can be viewed as a twoport network with $V_{\mathrm{p}}, I_{\mathrm{p}}$ as input variables and $V_{\mathrm{s}}, I_{\mathrm{s}}$ as output variables.

Here

$$
\begin{gathered}
z_{11}=\frac{1}{j \omega C_{\mathrm{p}}}+j \omega L_{\mathrm{p}}+R_{\mathrm{p}} \\
z_{12}=z_{21}=j \omega M \\
z_{22}=\frac{1}{j \omega C_{\mathrm{s}}}+j \omega L_{\mathrm{s}}+R_{\mathrm{s}} \\
I_{\mathrm{s}}^{\prime}=-I_{\mathrm{s}} .
\end{gathered}
$$

Using z-parameters, equations that describe the circuit in figure 6 can be written as follows:

$$
\begin{gathered}
V_{\mathrm{p}}=z_{11} I_{\mathrm{p}}+z_{12} I_{\mathrm{s}}^{\prime} \\
V_{\mathrm{s}}=z_{21} I_{\mathrm{p}}+z_{22} I_{\mathrm{s}}^{\prime} \\
V_{\mathrm{s}}=-I_{\mathrm{s}}^{\prime} R_{\mathrm{L}} .
\end{gathered}
$$

Solving Eqs. (8)-(10) gives voltage transfer characteristics $\left(V_{\mathrm{s}} / V_{\mathrm{p}}\right)$, current transfer characteristics $\left(I_{\mathrm{s}} / I_{\mathrm{p}}\right)$, input impedance characteristics $\left(V_{\mathrm{p}} / I_{\mathrm{p}}\right)$ and output current by input voltage characteristics $\left(I_{\mathrm{s}} / V_{\mathrm{p}}\right)$. These characteristics at any general frequency $\omega$ and at resonance frequency $\omega_{0}$ have been presented in table 3 .

From table 3 two important observations can be abstracted: first, one can observe that reflected impedance at resonance, i.e., ' $\frac{\omega_{o}^{2} M^{2}}{R_{\mathrm{s}}+R_{\mathrm{L}}}$ component in $Z_{\text {in }}$, is purely resistive. This means there is no VAR loading on primary by secondary, when secondary is perfectly tuned. Moreover the secondary operates at unity power factor.

Second, if winding resistance is neglected then secondary current $I_{s}$ is given by

$$
\left|I_{\mathrm{s}}\right|=\frac{V_{\mathrm{p}}}{\omega M}
$$

From Eq. (11) one can understand that when coils are fed from fixed-frequency constant-voltage supply then secondary current is independent of load and depends only on mutual inductance, i.e., SS topology is simply an equivalent ideal current source. This type of characteristics is useful in EV's battery charging application, for example in case of stationary charging or in-motion charging for guided motion such as monorail system [13]. Because of this property, SS topology has inherent short circuit protection. However this also means that output voltage cannot be regulated when the secondary is open circuited-a major drawback of this topology. 
Table 3. Characteristics of SS topology.

\begin{tabular}{lcc}
\hline Characteristics & At general frequency, $\omega$ & At resonance frequency $\omega_{\mathrm{o}}$ \\
\hline$h_{\mathrm{i}-\mathrm{ss}}=I_{\mathrm{S}} / I_{\mathrm{p}}$ & $\frac{z_{21}}{R_{\mathrm{L}}+z_{22}}$ & $\frac{j \omega_{\mathrm{o}} M}{R_{\mathrm{L}}+R_{s}}$ \\
$h_{\mathrm{v}-\mathrm{ss}}=V_{\mathrm{s}} / V_{\mathrm{p}}$ & $\frac{z_{21} R_{\mathrm{L}}}{R_{\mathrm{L}} z_{11}+z_{22} z_{11}-z_{12} z_{21}}$ & $\frac{j \omega_{\mathrm{o}} M R_{\mathrm{L}}}{R_{\mathrm{p}} R_{\mathrm{L}}+R_{\mathrm{s}} R_{\mathrm{p}}+\omega_{\mathrm{0}}^{2} M^{2}}$ \\
$Z_{\mathrm{in}}=V_{\mathrm{p}} / I_{\mathrm{p}}$ & $z_{11}-\frac{z_{12} z_{21}}{R_{\mathrm{L}}+z_{22}}$ & $R_{\mathrm{p}}+\frac{\omega_{\mathrm{o}}^{2} M^{2}}{R_{\mathrm{s}}+R_{\mathrm{L}}}$ \\
$I_{\mathrm{S}} / V_{\mathrm{p}}$ & $\frac{z_{21}}{R_{\mathrm{L}} z_{11}+z_{22} z_{11}-z_{12} z_{21}}$ & $\frac{j \omega_{0} M}{R_{\mathrm{p}} R_{\mathrm{L}}+R_{\mathrm{s}} R_{\mathrm{p}}+\omega_{\mathrm{o}}^{2} M^{2}}$ \\
\hline
\end{tabular}

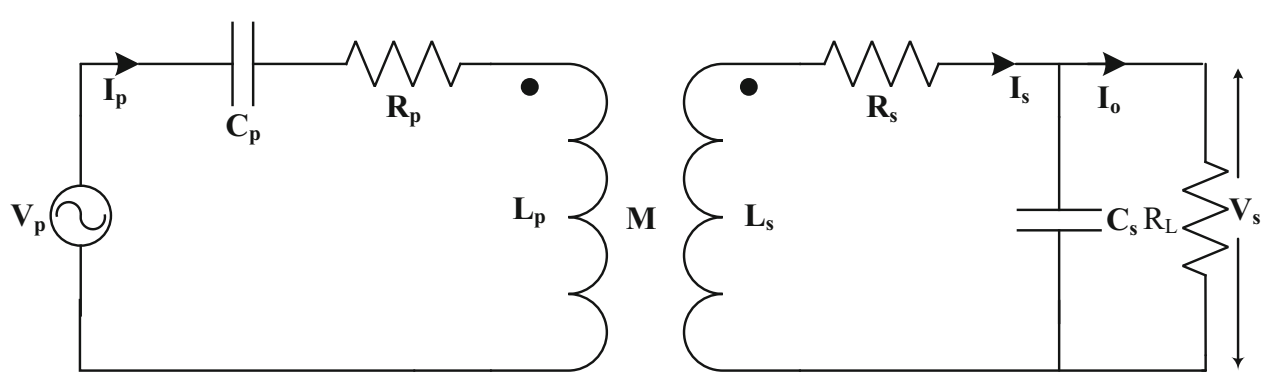

Figure 7. SP compensated IPT topology.

\section{Characteristics of SP topology}

Figure 7 gives the IPT coils with primary series and secondary parallel compensation.

Value of primary capacitance and secondary capacitance for SP topology at resonance frequency $\omega_{\mathrm{o}}$ is given by

$$
\begin{gathered}
C_{\mathrm{p}}=\frac{L_{\mathrm{s}} C_{\mathrm{s}}}{\left(L_{\mathrm{p}}-\frac{M^{2}}{L_{\mathrm{s}}}\right)} \\
C_{\mathrm{s}}=\frac{1}{\omega_{o}^{2} L_{\mathrm{s}}} .
\end{gathered}
$$

However, in the derivation of $C_{\mathrm{p}}$, effect of primary and secondary coil resistance has been neglected. Figure 8 gives the two-port model for SP topology shown in figure 7 in terms of $z$ parameters.

Here, $C_{1}$ and $R_{1}$ are series equivalent of parallel $C_{\mathrm{s}}$ and $R_{\mathrm{L}}$ and given by

$$
\begin{gathered}
R_{1}=\frac{R_{\mathrm{L}}}{1+\omega^{2} R_{\mathrm{L}}^{2} C_{\mathrm{s}}^{2}} \\
C_{1}=\frac{1+\omega^{2} R_{\mathrm{L}}^{2} C_{\mathrm{s}}^{2}}{\omega^{2} R_{\mathrm{L}}^{2} C_{\mathrm{s}}^{2}} C_{\mathrm{s}} .
\end{gathered}
$$

Other $z$ parameters are given as follows:

$$
\begin{gathered}
z_{11}=\frac{1}{j \omega C_{\mathrm{p}}}+j \omega L_{\mathrm{p}}+R_{\mathrm{p}} \\
z_{12}=z_{21}=j \omega M \\
z_{22}=j \omega L_{\mathrm{s}}+R_{\mathrm{S}}
\end{gathered}
$$

$$
\begin{gathered}
I_{\mathrm{s}}^{\prime}=-I_{\mathrm{s}} \\
z_{\mathrm{L}}=R_{1}+\frac{1}{j \omega C_{1}} .
\end{gathered}
$$

Using $z$-parameters, equations that describe the circuit in figure 8 can be written as follows:

$$
\begin{gathered}
V_{\mathrm{p}}=z_{11} I_{\mathrm{p}}+z_{12} I_{\mathrm{s}}^{\prime} \\
V_{\mathrm{s}}=z_{21} I_{\mathrm{p}}+z_{22} I_{\mathrm{s}}^{\prime} \\
V_{\mathrm{s}}=-I_{\mathrm{s}}^{\prime} Z_{\mathrm{L} .}
\end{gathered}
$$

Solving Eqs. (14)-(23) gives the characteristics of SP topology. These characteristics at any frequency $\omega$ and at resonance frequency $\omega_{\mathrm{o}}$ have been presented in table 4 .

When winding resistance is neglected, reflected impedance $\left(Z_{\mathrm{R}}\right)$ at resonance can be derived from $Z_{\text {in }}$ expression in table 4 and is given by

$$
Z_{\mathrm{R}}=\frac{M^{2} R_{\mathrm{L}}}{L_{\mathrm{s}}^{2}}-\frac{j \omega_{o} M^{2}}{L_{\mathrm{s}}} .
$$

From Eq. (24) one can observe that reflected impedance is not purely resistive but rather consists of a load-independent capacitive reactance. This means secondary is not operating at unity power factor at $\omega_{\mathrm{o}}$ as opposed to SS topology discussed previously. Moreover this capacitive reactance will oppose the primary inductance $L_{\mathrm{p}}$, causing a reduction in $\mathrm{Z}_{\text {in. }}$ This means a larger capacitor will be required to tune out the additional VAR loading on the primary by secondary coil. This can also be justified by comparing Eqs. (2) and (12). 


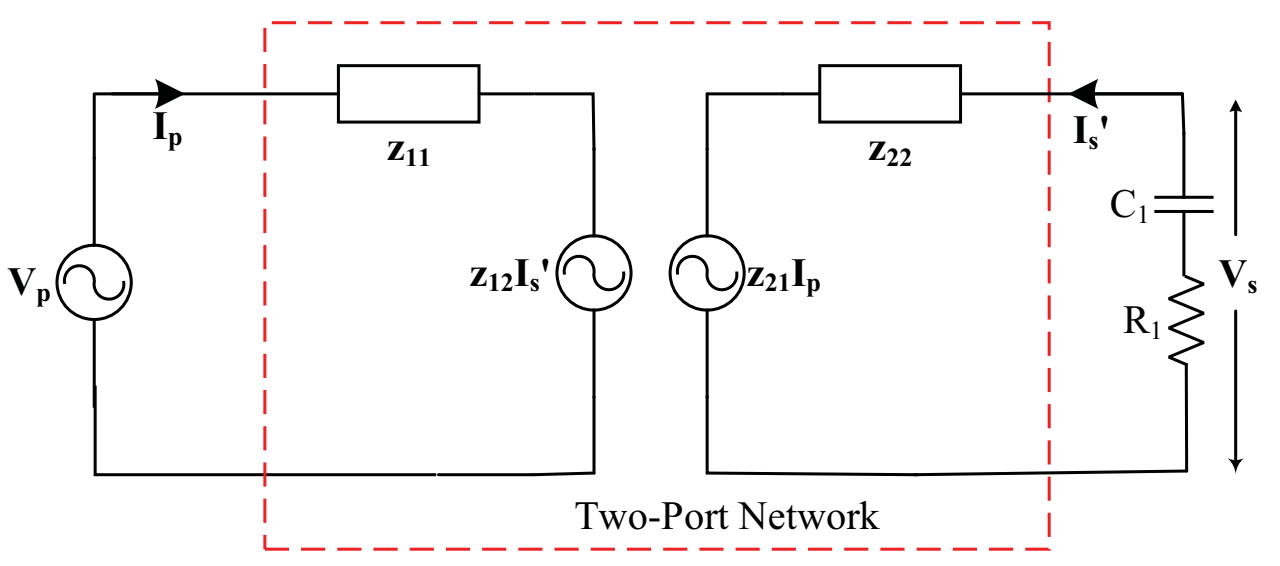

Figure 8. Two-port model of SP topology.

Table 4. Characteristics of SP topology.

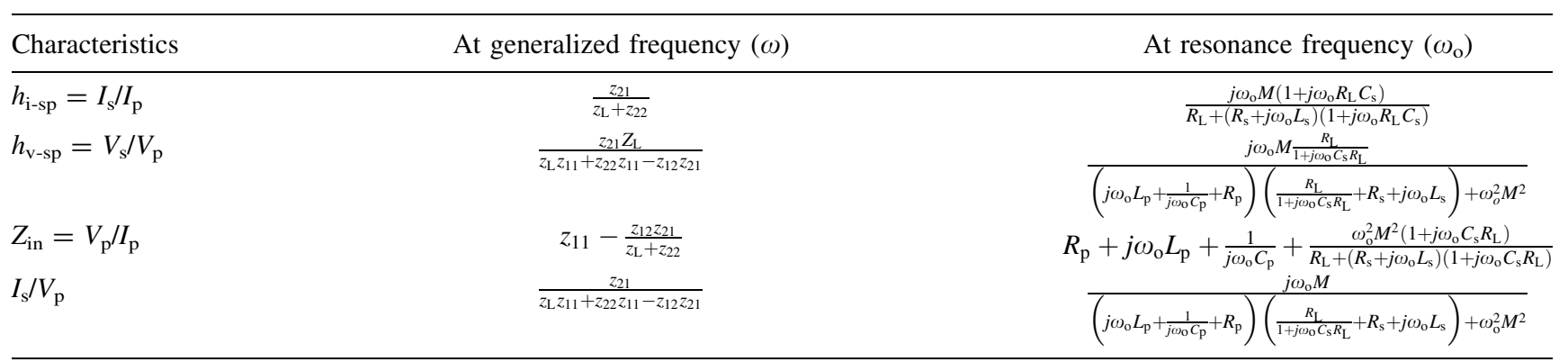

After neglecting winding resistance and using Eqs. (12) and (13), voltage transfer characteristics of SP topology at resonance frequency can be simplified as

$$
h_{\mathrm{v}-\mathrm{sp}}=\frac{V_{\mathrm{s}}}{V_{\mathrm{p}}}=\frac{L_{\mathrm{s}}}{M} \text {. }
$$

From Eq. (25) one can observe that output voltage is independent of load $R_{\mathrm{L}}$ and will be constant as long as mutual inductance $M$ does not vary and therefore SP topology acts as an ideal voltage source. Constant voltage characteristics are useful in multiple outlets systems, systems that have intermediate dc bus such as modern variable speed ac drives or for multiple outlets. With a constant input voltage source of fixed frequency, SP topology can also be used for stationary or inmotion EV battery charging if a switched mode controller is included in the pickup that can match the current requirement for battery charging. As opposed to SS topology, SP topology is not short circuit proof.

\section{Simulation and experimental results}

To verify the current-source characteristics of SS topology and voltage-source characteristics of SP topology the SS and SP topologies were simulated in MATLAB and simulation results were experimentally verified. Figure 9 gives the circuit diagram for the circuit used in simulation and experiment.

Table 5 gives the specification of SS and SP topologies for simulation circuit.

Figure 10 gives the set-up for experiment. For the experiment, compensation capacitors with very low ESR polypropylene film type have been selected.

\subsection{Current source characteristics of SS topology}

To verify that SS topology behaves as a current source when fed from constant-voltage fixed-frequency supply, load resistance was varied in the sequence $(1.24,2.24,3.49$, $4.33,5.35$ and 6.22) $\Omega$ while dc link voltage of inverter was fixed at $25 \mathrm{~V}$ and switching frequency was kept constant at $20 \mathrm{kHz}$. Same values of load resistance were used in simulation. Theoretical value of capacitors shown in table 5 has been used in the simulation but due to practical availability, value of capacitor used in experimental set-up was adjusted to $C_{\mathrm{p}}=0.445 \mu \mathrm{F}$ and $C_{\mathrm{s}}=2.7 \mu \mathrm{F}$. Secondary current by input voltage $\left(I_{\mathrm{s}} / V_{\mathrm{p}}\right)$ for simulation and experiment has been plotted in figure 11 .

From figure 11 one can observe that in simulation $I_{\mathrm{s}} / V_{\mathrm{p}}$ is almost constant irrespective of the load variation. A fine 


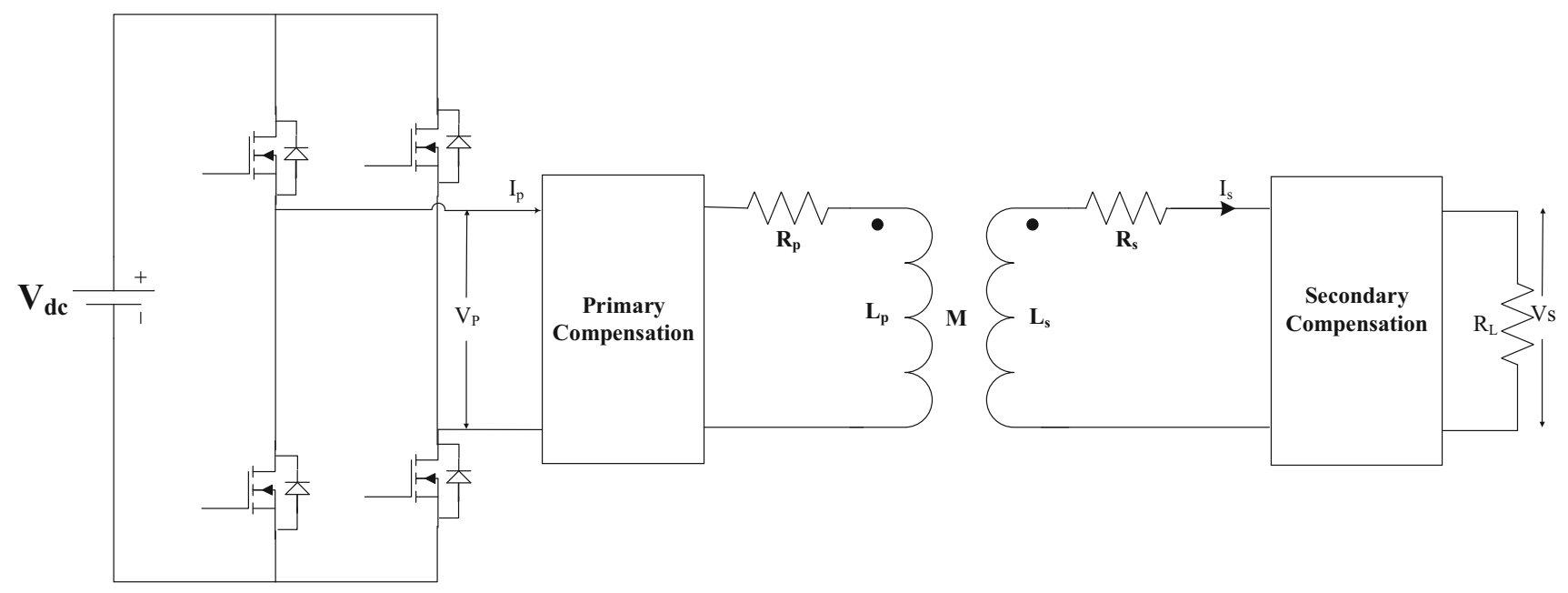

Figure 9. Circuit diagram for simulation and experiment.

Table 5. Specification of circuit for simulation.

\begin{tabular}{lc}
\hline Circuit specification for SS topology & \\
$V_{\mathrm{dc}}$ & $25 \mathrm{~V}$ \\
$C_{\mathrm{p}}$ & $0.44558 \mu \mathrm{F}$ \\
$C_{\mathrm{s}}$ & $2.8461 \mu \mathrm{F}$ \\
$f_{\mathrm{o}}=f_{\mathrm{sw}}$ & $20 \mathrm{kHz}$ \\
Circuit specification for SP topology & \\
$V_{\mathrm{dc}}$ & $15 \mathrm{~V}$ \\
$C_{\mathrm{p}}$ & $0.56026 \mu \mathrm{F}$ \\
$C_{\mathrm{s}}$ & $2.468 \mu \mathrm{F}$ \\
$f_{\mathrm{o}}=f_{\mathrm{sw}}$ & $21.5 \mathrm{kHz}$ \\
\hline
\end{tabular}

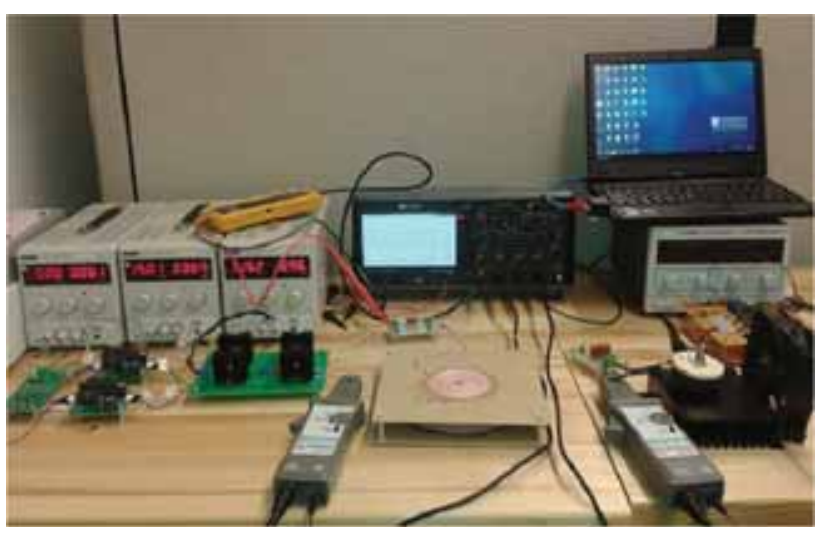

Figure 10. Experimental set-up.

linear drop is due to winding resistance. In experiment, drop is more because of switching losses, coil resistance and resistance of the connecting wire. Moreover, connecting wires were normal stranded wire rather than litz wire. At $20 \mathrm{kHz}$ such wire behaves as an inductor; hence tuning deviates from ideal condition. However for Li-ion battery, the constant current does not need to be precise and a semi-

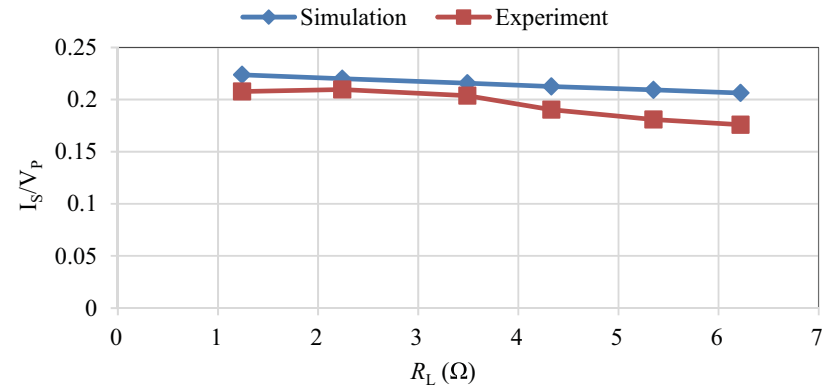

Figure 11. $I_{\mathrm{S}} / V_{\mathrm{p}}$ versus load resistance $\left(R_{\mathrm{L}}\right)$ variations for $\mathrm{SS}$ topology.

constant current is allowed. Often, in linear chargers, the current is ramped up as the cell voltage rises in order to minimize the heat dissipation of the pass transistor.

\subsection{Voltage source characteristics of SP topology}

To show that SP topology acts as a voltage source when fed from constant-voltage and fixed-frequency supply, load was varied in the sequence $(1.2,2.21,3.45,4.57,5.34$ and 6.23) $\Omega$ while dc link of inverter was fixed at $15 \mathrm{~V}$ and switching frequency was kept constant at $21.5 \mathrm{kHz}$. Due to practical availability, values of capacitors used in experimental setup were adjusted to $C_{\mathrm{p}}=0.56 \mu \mathrm{F}$ and $C_{\mathrm{s}}=2.7 \mu \mathrm{F}$. Voltage transfer characteristics $\left(V_{\mathrm{s}} / V_{\mathrm{p}}\right)$ for simulation and experiment have been plotted in figure 12 .

From figure 12 one can observe that voltage transfer characteristics obtained with experiment matched well with the simulation results. A slight discrepancy in the characteristics was observed, which is partially due to parasitic resistances of coils and connecting wires and partially due to absence of perfect tuning in secondary winding. One can observe that $V_{\mathrm{s}} / V_{\mathrm{p}}$ ratio is almost independent of load, which gives voltage-source characteristics to the SP topology. 


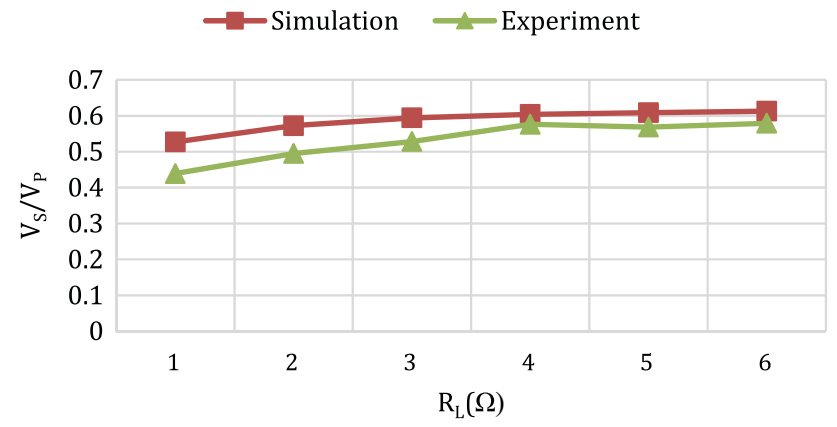

Figure 12. $V_{\mathrm{s}} / V_{\mathrm{p}}$ versus load resistance $\left(R_{\mathrm{L}}\right)$ variations for $\mathrm{SP}$ topology.

\section{Conclusion}

In this paper, behaviour of SS and SP topologies when fed from constant-voltage fixed-frequency supply has been studied. SS topology was found to have constant-current (CC) output characteristics whereas SP topology gives constant-voltage (CV) output. To simplify the analysis, the concept of two-port network has been utilized for deriving the characteristics of circuits. Theory has been validated using MATLAB simulation and hardware implementation. Since CC and CV are the main charging stages, either of the topologies can be used for EV battery charging applications. Since SS topology acts as a voltage source it requires a rectifier with capacitive filter on secondary side; however, SP topology, due to its CV output, will require rectifier with LC output filter. In order to reduce the weight and volume of the secondary side a capacitor filter is preferable [16]. Moreover, from Eq. (12), primary compensation in SP topology depends upon mutual inductance, which tends to vary with airgap and misalignment. Based on this discussion, SS topology is recommended for EV battery charging application. Characteristics not only establish the concept about circuit but also help in selection of a particular topology for particular application.

\section{Nomenclature}

$V_{\mathrm{p}} \quad$ Primary voltage

$L_{\mathrm{p}} \quad$ Primary self-inductance

$C_{\mathrm{p}} \quad$ Primary capacitor

$L_{\mathrm{s}} \quad$ Secondary self-inductance

$C_{\mathrm{S}} \quad$ Secondary capacitor

$R_{\mathrm{L}} \quad$ Load resistance

$I_{p} \quad$ Primary current

$I_{s} \quad$ Secondary current

$I_{\mathrm{O}} \quad$ Output current

$V_{\mathrm{s}} \quad$ Secondary voltage

hi-sp Current transfer ratio of SP topology

hv-sp Voltage transfer ratio of SP topology

$Z_{\text {in }} \quad$ Total input impedance seen by source hi-ss Current transfer ratio of SS topology

hv-ss Voltage transfer ratio of SS topology

$f_{\mathrm{o}} \quad$ Resonant frequency

$f_{\mathrm{sw}} \quad$ Switching frequency

$\omega \quad$ Angular frequency

$\omega_{\mathrm{o}} \quad$ Angular resonant frequency

\section{References}

[1] Budhia M, Boys J, Covic G and Huang C 2013 Development of a single sided flux magnetic coupler for electric vehicle IPT charging systems. IEEE Trans. Ind. Electron. 60(1): 318-328

[2] Aditya K and Williamson S 2014 Design considerations for loosely coupled inductive power transfer (IPT) system for electric vehicle battery charging - a comprehensive review. In: Proceedings of the Transportation Electrification Conference and Expo (ITEC), pp. 1-6

[3] Aditya K and Williamson S 2014 Comparative study of seriesseries and series-parallel topology for long track EV charging application. In: Proceedings of the IEEE Transportation Electrification Conference and Expo (ITEC), pp. 1-5

[4] Covic G A and Boys J T 2013 Inductive power transfer. Proc. IEEE 101(6): 1-14

[5] Laouamer R, Bmnello M, Ferrieux J P, Normand O, Buchheit N 1997 A multi-resonant converter for non-contact charging with electromagnetic coupling. In: Proceedings of International Conference on Industrial Electronics, Control and Instrumentation, pp. 792-797

[6] Wang C S, Stielau O H and Covic G A 2005 Design considerations for a contactless electric vehicle battery charger. IEEE Trans. Ind. Electron. 52(5): 1308-1314

[7] Imura T 2010 Study on maximum air-gap and efficiency of magnetic resonant coupling for wireless power transfer using equivalent circuit. In: Proceedings of the IEEE International Symposium on Industrial Electronics, pp. 3664-3669

[8] Tesla N 1892 Experiments with alternate currents of high potential and high frequency. J. Inst. Electr. Eng. 21(97): 51

[9] Pinuela M, Yates D C, Lucyszyn S and Mitcheson P D 2013 Maximizing dc-to-load efficiency for inductive power transfer. IEEE Trans. Power Electron. 28(5): 2437-2447

[10] Wang C S, Covic G A and Stielau O H 2004 Power transfer capability and bifurcation phenomena of loosely coupled inductive power transfer systems. IEEE Trans. Ind. Electron. 51(1): 148-156

[11] Huh J, Lee S W, Lee W Y, Cho G H and Rim C T 2011 Narrow width inductive power transfer system for online electrical vehicles. IEEE Trans. Power Electron. 26(12): 3666-3679

[12] Khaligh A and Li Z 2010 Battery, ultracapacitor, fuel cell, and hybrid energy storage systems for electric, hybrid electric, fuel cell, and plug-in hybrid electric vehicles: state of the art. IEEE Trans. Veh. Technol. 59(6): 2806-2814

[13] Weixiang A, Thanh T V and Kapoor A 2012 Charging algorithms of lithium-ion batteries: an overview. In: Proceedings of the IEEE Conference on Industrial Electronics and Applications (ICIEA), pp. 1567-1572 
[14] Eghtesadi M 1990 Inductive power transfer to an electric vehicle-analytical model. In: Proceedings of the IEEE Conference on Vehicular Technology, pp. 100104

[15] Stielau O H and Covic G A 2000 Design of loosely coupled inductive power transfer systems. In: Proceedings of the
IEEE 2000 International Conference on Power System Technology, pp. 85-90

[16] Kutkut N H, Divan D M, Novotny D W and Marion R H 1998 Design considerations and topology selection for a 120-kW IGBT converter for EV fast charging. IEEE Trans. Power Electron. 13(1): 169-178 